\title{
Boolean lattices: Ramsey properties and embeddings.
}

\author{
Maria Axenovich and Stefan Walzer *
}

August 23, 2021

\begin{abstract}
A subposet $Q^{\prime}$ of a poset $Q$ is a copy of a poset $P$ if there is a bijection $f$ between elements of $P$ and $Q^{\prime}$ such that $x \leq y$ in $P$ iff $f(x) \leq f(y)$ in $Q^{\prime}$. For posets $P, P^{\prime}$, let the poset Ramsey number $R\left(P, P^{\prime}\right)$ be the smallest $N$ such that no matter how the elements of the Boolean lattice $Q_{N}$ are colored red and blue, there is a copy of $P$ with all red elements or a copy of $P^{\prime}$ with all blue elements. We provide some general bounds on $R\left(P, P^{\prime}\right)$ and focus on the situation when $P$ and $P^{\prime}$ are both Boolean lattices. In addition, we give asymptotically tight bounds for the number of copies of $Q_{n}$ in $Q_{N}$ and for a multicolor version of a poset Ramsey number.
\end{abstract}

\section{Introduction}

A classical hypergraph Ramsey number $R(G, H)$ for $k$-uniform hypergraphs $G$ and $H$ is the smallest $n$ such that any red/blue edge-coloring of a complete $k$-uniform hypergraph on $n$ vertices contains a red copy of $G$ or a blue copy of $H$. The existence of this number was proved by Ramsey [18] in 1930, but the problem of determining these and "multicolor" numbers remains open and generates a lot of research activity, see for example $[5,4,3,6,1,11]$.

While classical Ramsey theory provides results about unavoidable uniform set systems such as $k$-uniform hypergraphs, here we investigate Ramsey numbers for set systems with various set sizes treated as partially ordered sets.

A partially ordered set, a poset, is a set with an accompanying relation " $\leq$ " that is transitive, reflexive, and antisymmetric. A Boolean lattice of dimension $n$, denoted $Q_{n}$, is the power set of an $n$-element ground set $X$ equipped with inclusion relation, we also write $2^{X}$ for such a lattice. The $k$-th layer of $Q_{n}$ is the set of all $k$-element subsets of the ground set, $0 \leq k \leq n$. An injective mapping $f: P \rightarrow P^{\prime}$ is an embedding of a poset $P$ into another poset $P^{\prime}$ if for any $A, B \in P$ we have $A \leq B$ in $P$ iff $f(A) \leq f(B)$ in $P^{\prime}$. In this case, we say that $f(P)$ is a copy of $P$ in $P^{\prime}$. The 2-dimension of a poset $P$, defined by Trotter [20] and denoted by $\operatorname{dim}_{2}(P)$, is the smallest $n$ such that $Q_{n}$ contains a copy of $P$. An $n$-element antichain $A_{n}$ is a poset with any two elements not comparable, an $n$-element chain $C_{n}$ is a poset with any two elements comparable.

A very general problem of determining what posets have Ramsey property was solved by Nešetřil and Rödl [15]. Here, a poset $X$ has Ramsey property if for any poset $P$ there is a poset $Z$ such that when one colors the copies of $X$ in $Z$ red or blue, there is a copy of $P$ in $Z$ such that all copies of $X$ in this copy of $P$ are red or all of them are blue. In the current paper, $X$ is always the single-element poset, i.e., the elements of posets are colored, instead of more complicated substructures. In that case, the existence of $Z$ is guaranteed, so we direct our attention to quantitative aspects and the asymmetric case where the two colors are associated with different posets.

${ }^{*}$ Karlsruhe Institute of Technology 
Let $P$ and $P^{\prime}$ be two posets and $g$ be a poset parameter, then $R_{g}\left(P, P^{\prime}\right)$ is the smallest $g^{\prime}$ such that there is a poset $Z$ with $g(Z)=g^{\prime}$ and such that in any red/blue coloring of $Z$ there is a copy of $P$ with all red elements or a copy of $P^{\prime}$ with all blue elements. Kierstead and Trotter [9] considered $g$ to be width, height, or size, see also $[21,19,16]$. In this paper we initiate the study of Ramsey numbers for posets that extends the previously defined notions and fits the concept of extremal functions of posets $[17,13]$.

For posets $P$ and $P^{\prime}$, let the poset Ramsey number $R_{\operatorname{dim}_{2}}\left(P, P^{\prime}\right)$ be the smallest $N$ such that any red/blue-coloring of $Q_{N}$ contains either a red copy of $P$ or a blue copy of $P^{\prime}$. We use simply $R(P, Q)$ instead of $R_{\operatorname{dim}_{2}}(P, Q)$ when it is clear from context.

For example, $R\left(C_{n}, C_{n}\right)=2 n-2$ for a chain $C_{n}$. Indeed, in a 2-colored $Q_{2 n-2}$ there is a chain with $2 n-1$ elements, $n$ of those have the same color and form a copy of $C_{n}$; a coloring of $Q_{2 n-3}$ with $n-1$ layers in one color and $n-1$ layers in another color has no monochromatic copy of $C_{n}$. Another example is $R\left(A_{n}, A_{n}\right)=\min \left\{N: 2 n-1 \leq{ }_{\lfloor N / 2\rfloor}\left(\begin{array}{c}N \\ \lfloor N\end{array}\right)\right.$, for an antichain $A_{n}$. To see that, observe that at least half of the elements in the middle layer in a 2-colored $Q_{N}$ have the same color and there are at least $n$ of them. On the other hand, partition the elements of $Q_{N-1}$ into at most $2 n-2$ chains. Such a partition exists, see for example [12]. Color $n-1$ chains red and the remaining (at most $n-1$ ) chains blue. Since each antichain has at most one element from each of these chains, there is no monochromatic antichain with $n$ elements. The focus of this paper is the case when $P$ and $P^{\prime}$ are Boolean lattices themselves, i.e., $R\left(Q_{n}, Q_{m}\right)$.

Theorem 1. For any integers $n, m \geq 1$,

(i) $2 n \leq R\left(Q_{n}, Q_{n}\right) \leq n^{2}+2 n$,

(ii) $R\left(Q_{1}, Q_{n}\right)=n+1$,

(iii) $R\left(Q_{2}, Q_{n}\right) \leq 2 n+2$,

(iv) $R\left(Q_{n}, Q_{m}\right) \leq m n+n+m$,

(v) $R\left(Q_{2}, Q_{2}\right)=4, R\left(Q_{3}, Q_{3}\right) \in\{7,8\}$,

(vi) A Boolean lattice $Q_{3 n \log (n)}$ whose elements are colored red or blue randomly and independently with equal probability contains a monochromatic copy of $Q_{n}$ asymptotically almost surely.

We prove a more general result in terms of the 2-dimension and height $h$ of a poset $P$, where $h(P)$ is the number of elements in a largest chain of $P$. The lexicographic product $P \times Q$ of two posets is defined by taking a disjoint union of $|P|$ copies of $Q$ and making all elements in $i$ th copy less than each element in $j$ th copy iff the $i$ th element of $P$ is less than $j$ th element of $P$. Formally, the set of elements of $P \times Q$ is a cartesian product of elements of $P$ and $Q$ and $\left(p_{1}, q_{1}\right) \leq\left(p_{2}, q_{2}\right)$ in $P \times Q$ iff $p_{1} \leq p_{2}$ or $\left(p_{1}=p_{2}\right.$ and $\left.q_{1} \leq q_{2}\right)$. Note that the product operation is not symmetric, i.e., in general $P \times Q$ and $Q \times P$ are not isomorphic. We say that a coloring of $2^{S}$ is layered for a set $S$ if for each $i$, $i=0, \ldots,|S|$ all subsets of $S$ of size $i$ have the same color.

Lemma 2 (Layered Lemma). For any $n$ there is $N$ such that in any red/blue coloring $c$ of subsets of $[N]$ there is a set $S \subseteq[N]$ such that $|S|=n$ and $c$ is layered on $2^{S}$.

Lemma 3 (Blob Lemma). Let $P$ be a poset and $m \geq 1$ be an integer. If $N=\operatorname{dim}_{2}(P)+h(P) m$, then $Q_{N}$ contains a copy of $P \times Q_{m}$. In particular, $R\left(P, Q_{m}\right) \leq \operatorname{dim}_{2}(P)+h(P) m$.

Lemma 4 (Antichain Lemma). If in a red/blue coloring of $2^{[N]}$ the red elements form antichains $\mathcal{A}_{1}, \ldots, \mathcal{A}_{\ell}, \ell<N$, such that $A_{i}$ is a set of minimal elements in $\mathcal{A}_{i} \cup \cdots \cup \mathcal{A}_{\ell}, i=1, \ldots, \ell$, then there is a blue copy of $2^{[N-\ell]}$. 
We give some more definitions and prove Lemmas 2, 3, 4 in Section 2. We prove Theorem 1 in Section 3.

In studying Ramsey properties of posets, specifically Boolean lattices, we prove a result of independent interest concerning embeddings of $Q_{n}$ into $Q_{N}$. We describe a bijective mapping between the set of such embeddings and a set of some special sequences in Section 4. As a corollary, we determine the number of such embeddings.

Theorem 5. Let $n \leq N$. The number $e(n, N)$ of embeddings of $Q_{n}$ into $Q_{N}$ satisfies the inequality

$$
\frac{N !}{(N-n) !}(a(n)-n)^{N-n} \leq e(n, N) \leq \frac{N !}{(N-n) !} a(n)^{N-n},
$$

where $a(n)$ is the number of distinct antichains in $2^{[n]}$. In particular

$$
e(n, N)=2^{\left(\left\lfloor\frac{n}{2}\right\rfloor\right)(N-n)(1+o(1))} .
$$

The multicolor Ramsey number for a poset $P$ is defined, for a given $k$, to be the smallest integer $n$ such that any coloring of $Q_{n}$ in $k$ colors contains a copy of $P$ in one of the colors. This number is denoted by $R_{k}(P)$. The following theorem is proved in Section 5 .

Theorem 6. For any poset $P$ that is not an antichain, $R_{k}(P)=\Theta(k)$.

Finally, we make a quick comparison between Ramsey numbers for Boolean lattices and Ramsey numbers for Boolean algebras. A Boolean algebra $B_{n}$ of dimension $n$ is a set system $\left\{X_{0} \cup \bigcup_{i \in I} X_{i}\right.$ : $I \subseteq[n]\}$, where $X_{0}, X_{1}, \ldots, X_{n}$ are pairwise disjoint sets, $X_{i} \neq \emptyset$ for $i=1, \ldots, n$. To see the difference between Boolean algebras and copies of $Q_{n}$ consider three families of sets in $2^{[6]}: \mathcal{F}_{1}=\{\{2\},\{2,3\}$, $\{2,4,5\},\{2,3,4,5,6\}\}, \mathcal{F}_{2}=\{\{2\},\{2,3,4\},\{2,5\},\{2,3,4,5\}\}, \mathcal{F}_{3}=\{\{2\},\{2,3\},\{2,3,5\},\{2,3,4,5\}\}$. Here $\mathcal{F}_{2}$ is a Boolean algebra of dimension 2 with $X_{0}=\{2\}, X_{1}=\{3,4\}, X_{2}=\{5\}$, but $\mathcal{F}_{1}$ and $\mathcal{F}_{3}$ are not. The families $\mathcal{F}_{1}$ and $\mathcal{F}_{2}$ are copies of $Q_{2}$, however $\mathcal{F}_{3}$ is not a copy of $Q_{2}$ because in $Q_{2}$ there are two incomparable elements and in $\mathcal{F}_{3}$ any two elements are comparable. Boolean algebras have much more restrictive structure than Boolean lattices. If a subset of $Q_{N}$ contains a Boolean algebra of dimension $n$, then it clearly has a copy of $Q_{n}$, but not necessarily the other way around. Gunderson, Rödl, and Sidorenko [7] considered the number $R_{\text {Alg }}(n)$, defined to be the smallest $N$ such that any red/blue coloring of subsets of an $N$ element set contains a red or a blue Boolean algebra of dimension $n$. Note that here "contains" means not a subposet containment but simply a subset containment in $2^{[N]}$. The existence of $R_{\mathrm{Alg}}(n)$ and thus of $R_{\mathrm{dim}_{2}}\left(Q_{n}, Q_{n}\right)$ easily follows from Lemma 2. However, the bounds given by this approach are very large. We state the bounds on $R_{\mathrm{Alg}}(n)$, here the lower bound is given without a proof by Brown, Erdős, Chung, and Graham [2] and the upper bound is a recapturing of the arguments given by Gunderson, Rödl, and Sidorenko [7]. Here, $K^{n}(s, \ldots, s)$ is a complete $n$-uniform $n$-partite hypergraph with parts of size $s$ each and $R_{h}\left(K^{n}(2, \ldots, 2)\right)$ is be the smallest $N^{\prime}$ such that any 2-coloring of $K^{n}\left(N^{\prime}, \ldots, N^{\prime}\right)$ contains a monochromatic $K^{n}(2, \ldots, 2)$. Here $h$ stands for hypergraph.

Theorem 7. There is a positive constant c such that

$$
2^{c n} \leq R_{\text {Alg }}(n) \leq \min \left\{2^{2^{n+1} n \log n}, n R_{h}\left(K^{n}(2, \ldots, 2)\right)\right\} .
$$

We put the details of the upper bound in Section 6. The last section we devote to conclusions.

\section{Definitions and proofs of lemmas}

For a subset $U$ of elements in a poset $P$, the upper set of $U$ or simply the upset of $U$, is $U^{+}=\{x \in$ $P \mid \exists u \in U: u \leq x\}$. If $U=U^{+}$, then $U$ is called upper closed. We say that a sequence of sets is 
inclusion respecting if any higher indexed set is not a subset of a lower indexed set. Note that one can always create such an ordering for any family of sets by putting the sets of smaller cardinality ahead of sets of larger cardinality.

An affine cube, or a Hilbert cube of dimension $n$ is a set of integers $\left\{x_{0}+\sum_{i \in I} x_{i}: I \subseteq[n]\right\}$, where $x_{0}$ is a non-negative integer and $x_{1}, \ldots, x_{n}$ are positive integers. Note that if $\mathcal{A}=\left\{X_{0} \cup \bigcup_{i \in I} X_{i}: I \subseteq[n]\right\}$ is a Boolean algebra and $x_{i}=\left|X_{i}\right|, i=0, \ldots, n$, then the set of all sizes of sets occurring in $\mathcal{A}$ is equal to $\{|X|: X \in \mathcal{A}\}=\left\{x_{0}+\sum_{i \in I} x_{i}: I \subseteq[n]\right\}$ and therefore is a Hilbert cube. Hilbert [8] showed that for any positive integers $c$ and $n$ there is $N=h(n, c)$ such that any $c$-coloring of $[N]$ contains a monochromatic Hilbert cube of dimension $n$. Let $b(N, n)$ be the largest size of a family of subsets of $[N]$ that does not contain a Boolean algebra of dimension $n$.

The Lubell mass $\ell$ of a set family $\mathcal{F} \subseteq 2^{[N]}$ is $\ell(\mathcal{F})=\sum_{S \in \mathcal{F}}\left(\begin{array}{c}N \\ |S|\end{array}\right)^{-1}$. Note that $\ell\left(2^{[N]}\right)=N+1$. For a poset $P$, let $\lambda^{*}(P)=\lim \sup _{N \rightarrow \infty}\left\{\ell(\mathcal{F}): \mathcal{F} \subseteq 2^{[N]}\right.$ contains no copy of $\left.P\right\}$. Méroueh [14] proved that $\lambda^{*}(P)$ exists for any $P$.

For other definitions on graphs, posets, and set systems, we refer the reader to West and Trotter $[23,9]$.

Proof of Layered Lemma. Let $R^{k}(n)$ be the hypergraph Ramsey number that is equal to the smallest integer $N$ such that any red/blue coloring of $\left(\begin{array}{c}{[N]} \\ k\end{array}\right)$ contains a monochromatic complete hypergraph $\left(\begin{array}{l}S \\ k\end{array}\right)$ for $|S|=n$. Let $c$ be a red/blue coloring of $2^{[N]}$ for $N=R^{1}\left(R^{2}\left(R^{3}\left(\cdots\left(R^{n-1}(n)\right) \cdots\right)\right)\right)$. We shall prove by induction on $i, i=1, \ldots, n-1$ that there is a set $S_{i} \subseteq[N]$ such that $\left(\begin{array}{c}S_{i} \\ j\end{array}\right)$ is monochromatic for each $j=0, \ldots, i,\left|S_{i}\right|=R^{i+1}\left(R^{i+2}\left(\cdots\left(R^{n-1}(n)\right) \cdots\right)\right)$ ), and $S_{i} \supseteq S_{i+1}$. Since $N=R^{1}\left(R^{2}\left(R^{3}\left(\cdots\left(R^{n-1}(n)\right) \cdots\right)\right)\right)$, a coloring $c$ restricted to 1-element sets contains a monochromatic family of size $R^{2}\left(R^{3}\left(\cdots\left(R^{n-1}(n)\right) \cdots\right)\right)$. Let this set be $S_{1}$. Assume that $S_{1}, \ldots, S_{i-1}$ satisfying the assumption exist. Since $\left|S_{i-1}\right|=R^{i}\left(R^{i+1}\left(\cdots\left(R^{n-1}(n)\right) \cdots\right)\right.$ ), a coloring $c$ restricted to subsets of size $i$ in $S_{i-1}$ contains a set $S_{i} \subseteq S_{i-1}$ all of whose $i$-element subsets are of the same color and such that $\left|S_{i}\right|=R^{i+1}\left(R^{i+2}\left(\cdots\left(R^{n-1}(n)\right) \cdots\right)\right)$. This completes the induction. We have that $\left|S_{n-1}\right|=R^{n}(n)=n$, so $2^{S_{n-1}}$ is a Boolean lattice of dimension $n$ with a layered coloring.

Proof of Blob Lemma. Let $n=\operatorname{dim}_{2}(P)$ and let $f$ be an embedding of $P$ into $2^{[n]}$. Let $h(P)=h$ and $N=n+h m$. Consider a partition of $[N]=X_{0} \cup X_{1} \cup \ldots \cup X_{h}$, where $X_{0}=[n]$ and $\left|X_{i}\right|=m$, $i=1, \ldots, h$. Let $f_{i}$ be an isomorphism from $2^{[m]}$ to $2^{X_{i}}, i=1, \ldots, h$. We construct an embedding $g$ of $P \times 2^{[m]}$ into $2^{[N]}$. For $p \in P$ and $S \subseteq[m]$, let $h(p)$ be the number of elements in a longest chain in $P$ with maximum element $p$ and let $g((p, S))=f(p) \cup X_{1} \cup \ldots \cup X_{h(p)-1} \cup f_{h(p)}(S)$, where $h(p) \geq 1$.

By definition of the product, we have that $(p, S)$ is less than $\left(p^{\prime}, S^{\prime}\right)$ in $P \times 2^{[m]}$ iff $p$ is less than $p^{\prime}$ in $P$ or $\left(p=p^{\prime}\right.$ and $\left.S \subseteq S^{\prime}\right)$ and it is straightforward to check that $g$ respects this relation and is therefore an embedding.

For the second claim, consider a coloring of $2^{[\mathrm{N}]}$ in red and blue. Consider further a copy of $P \times 2^{[\mathrm{m}]}$ in it. If some copy of $2^{[m]}$ is blue, we are done, otherwise there is a red element $\left(p, S_{p}\right)$ for each $p \in P$ and some $S_{p} \subseteq[m]$. These red elements form a red copy of $P$.

Proof of Antichain Lemma. Consider $\mathcal{X}_{0}=2^{\{\ell+1, \ldots, N\}}$. Let $n=N-\ell$. We shall show a stronger statement by induction on $i, i=0, \ldots, \ell$ : there is a copy $\mathcal{X}_{i}$ of $2^{[n]}$ with sets from $\{1,2, \ldots, i, \ell+$ $1, \ldots, N\}$ such that this copy does not contain any set from $\mathcal{A}_{1}, \ldots, \mathcal{A}_{i}$.

Basis: The claim is trivial for $i=0$ since $\mathcal{X}_{0}$ fulfills the requirement as no sets are forbidden yet.

Step: Assume that $\mathcal{X}_{i-1}$ is a copy of $2^{[n]}$ consisting of subsets of $\{1,2, \ldots, i-1, \ell+1, \ldots, N\}$ and avoiding all of $\mathcal{A}_{1}, \mathcal{A}_{2}, \ldots, \mathcal{A}_{i-1}, i \geq 1$. Let $\mathcal{X}_{i}$ be the image of an embedding $f$ of $\mathcal{X}_{i-1}$, where $f(Y)=Y$ if $Y \notin \mathcal{A}_{i}^{+}, f(Y)=Y \cup\{i\}$ if $Y \in \mathcal{A}_{i}^{+}$. As before it is an embedding as it preserves inclusions. We only need to check that $f(Y) \notin \mathcal{A}_{1}, \mathcal{A}_{2}, \ldots, \mathcal{A}_{i}$ for any $Y \in \mathcal{X}_{i-1}$. Assume that $f(Y) \in \mathcal{A}_{j}$ for 
some $j \in\{1, \ldots, i\}$. Since any $Y$ from $\mathcal{X}_{i-1}$ is not in $\mathcal{A}_{1}, \ldots, \mathcal{A}_{i-1}$ and the elements of $\mathcal{A}_{i}$ in $\mathcal{X}_{i-1}$ were shifted up by $\{i\}$, we see that if $f(Y) \in \mathcal{A}_{j}$, then $Y \in \mathcal{A}_{i}^{+}$, and so $f(Y)=Y \cup\{i\}$. Thus, there is $B \in \mathcal{A}_{i}$, such that $B \subseteq Y$. So, $B \subseteq Y \subseteq Y \cup\{i\}=C$ for some $C \in \mathcal{A}_{j}$. If $i=j$ then we have two comparable sets $B$ and $C$ in an antichain $\mathcal{A}_{i}$, a contradiction. So, $j<i$. But $\mathcal{A}_{j}$ consists of minimal elements of $\mathcal{A}_{j} \cup \cdots \cup \mathcal{A}_{\ell}$ and $C \in \mathcal{A}_{j}$ is not a minimal element, a contradiction.

\section{Ramsey numbers for Boolean lattices}

Proof of Theorem 1 (i). For the lower bound on $R\left(Q_{n}, Q_{n}\right)$, consider $Q_{2 n-1}$. Color the sets of sizes $0, \ldots, n-1$ red and all other sets blue. Then there is no monochromatic chain with $n+1$ elements and thus there is no monochromatic copy of $Q_{n}$.

For the upper bound on $R\left(Q_{n}, Q_{n}\right)$, consider a red/blue coloring of $Q_{n^{2}+2 n}$. Let the ground set be $X_{0} \cup X_{1} \cup \ldots \cup X_{n+1}$, where $X_{i}$ 's are pairwise disjoint and of size $n$ each. Consider families of sets $\mathcal{B}_{Y}$ for each $Y \subseteq X_{0}$ with $|Y| \geq 1$ to be $\mathcal{B}_{Y}=\left\{Y \cup X_{1} \cup \ldots \cup X_{|Y|} \cup X: X \subseteq X_{|Y|+1}\right\}$, let $\mathcal{B}_{\emptyset}=2^{X_{1}}$. We see that each $\mathcal{B}_{Y}$ is a copy of $Q_{n}$. If this copy is blue, then $\mathcal{B}_{Y}$ gives a monochromatic copy of $Q_{n}$. Otherwise, there is a red element in each $\mathcal{B}_{Y}$. This element is $Z_{Y}=Y \cup X_{1} \cup \ldots \cup X_{|Y|} \cup S_{Y}$, where $S_{Y} \subseteq X_{|Y|+1}$. We claim that these elements form a red copy of $Q_{n}$. Indeed, we see for $Y, Y^{\prime} \subseteq[n]$ that $Y \subseteq Y^{\prime}$ iff $Z_{Y} \subseteq Z_{Y^{\prime}}$.

Proof of Theorem 1 (ii). To show that $R\left(Q_{1}, Q_{n}\right)=n+1$, consider a red/blue coloring of $Q_{n+1}$. If there are two red sets $A \subseteq B$, then they form a red copy of $Q_{1}$ and we are done. So, we can assume that the red sets form an antichain, $\mathcal{A}$. From Lemma 4 we see that there is a copy of $Q_{n}$ avoiding $\mathcal{A}$. To see that $R\left(Q_{1}, Q_{n}\right)>n$, color one element of $Q_{n}$ red and other blue.

Proof of Theorem 1 (iii). To show that $R\left(Q_{2}, Q_{n}\right) \leq 2 n+2$, consider a red/blue coloring of $Q_{2 n+2}$. Consider the poset formed by red elements. Assume that it has height at least $n+3$. Then there are two sets $A \subseteq B,|B \backslash A| \geq n+2$. Let $\mathcal{X}=\{Y: A \subseteq Y \subseteq B\}$. Then $\mathcal{X}$ is a copy of $Q_{m}, m \geq n+2$, with maximal and minimal elements red. This implies that all red elements in this poset form a chain, otherwise there is a red copy of $Q_{2}$. Let $a$ be contained in all sets of this chain but not in $A$. Let $b \in B \backslash(A \cup\{a\})$. Consider $\mathcal{X}^{\prime}=\{Y \in \mathcal{X}: a \notin Y, b \in Y\}$. Then $\mathcal{X}^{\prime}$ is a blue copy of $Q_{m-2}$ containing a blue copy of $Q_{n}$. Thus, we can assume that the height $h$ of the red poset $R$ is at most $n+2$. Build an antichain $\mathcal{A}_{1}$ to be the set of minimal elements of $R$ and an antichain $\mathcal{A}_{i}, i>1$ to be the set of minimal elements of $R \backslash\left(\mathcal{A}_{1} \cup \cdots \cup \mathcal{A}_{i-1}\right), i=1, \ldots, h$. Applying Lemma 4 , we see that there is a blue copy of $Q_{2 n+2-(n+2)}=Q_{n}$.

Proof of Theorem 1 (iv). The bound on $R\left(Q_{n}, Q_{m}\right)$ is a corollary of the Blob Lemma. Note that our upper bound on $R\left(Q_{n}, Q_{n}\right)$ also follows from the Blob Lemma, but we wrote an explicit direct proof.

Proof of Theorem $1(v)$. To prove that $R\left(Q_{2}, Q_{2}\right) \geq 4$, consider a layered coloring of $Q_{3}$ with two red layers and two blue layers. This coloring has no monochromatic copy of $Q_{2}$. For the upper bound, consider a red/blue coloring of $2^{[4]}$ and treat two cases: when $\emptyset$ and [4] have the same or different colors. Relatively easy case analysis shows that in both cases there is a monochromatic copy of $Q_{2}$.

To prove that $R\left(Q_{3}, Q_{3}\right) \in\{7,8\}$ we first give an explicit coloring of $2^{[6]}$ containing no monochromatic copy of $Q_{3}$. We list the family $\mathcal{R}$ of red sets in Figure 1. All other sets are blue, denote their family by $\mathcal{B}$. Note that a set $S$ is in $\mathcal{R}$ iff $[6] \backslash S$ is in $\mathcal{B}$. So, it is sufficient to verify that $\mathcal{R}$ contains no copy of $Q_{3}$. 


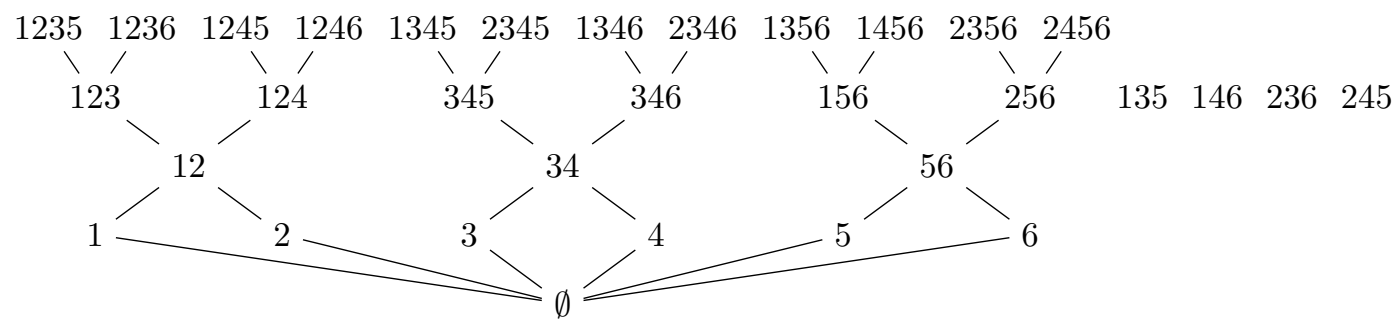

Figure 1: The family $\mathcal{R}$ of red subsets of [6] that avoid a copy of $Q_{3}$. Braces and commas are omitted. The lines illustrate inclusion, but inclusions involving the four sets on the right are ommitted to keep the picture simple.

Assume for the contrary that $f: 2^{[3]} \rightarrow \mathcal{R}$ is an embedding. Let $Y=f([3])$. Clearly, $Y$ should have size 3 or 4 . We assume that $|Y|=4$, since replacing $Y$ with one of its superset in $\mathcal{R}$ still gives a copy of $Q_{3}$. Without loss of generality $Y=\{1,2,3,5\}$. Then $2^{Y} \cap \mathcal{R}=\{\{1,2,3,5\},\{1,2,3\},\{1,3,5\}$, $\{1,2\},\{1\},\{2\},\{3\},\{5\}, \emptyset\}$, that clearly does not contain a copy of $Q_{3}$.

To show that $R\left(Q_{3}, Q_{3}\right) \leq 8$, consider a red/blue coloring of $2^{[8]}$. We say that a family of five distinct sets $A_{1}, \ldots, A_{5}$ forms a configuration $\mathcal{A}$ if either $A_{1}, \ldots, A_{5}$ or their complements satisfy the following conditions: $A_{1} \subset A_{2}, A_{3}, A_{4} \subset A_{5},\left|A_{5} \backslash A_{i}\right| \geq 5$ for $i=2,3,4,\left|A_{2}\right|=\left|A_{3}\right|=\left|A_{4}\right|$, and $\left|A_{i} \cup A_{j}\right|=\left|A_{i}\right|+1,2 \leq i<j \leq 4$. We treat four cases and in each of them we find either a monochromatic copy of $Q_{3}$ or a monochromatic family $\mathcal{A}$.

Case 1. The elements of some four layers have the same color. Then there is a monochromatic copy of $Q_{3}$.

Case 2. The elements of some three consecutive layers are of the same color, say red, and Case 1 does not hold. If there is a red element above or below the three red layers, then this element and some 7 members of the three red layers form a red copy of $Q_{3}$. If there is no such element, then there are 6 blue layers, that brings us to Case 1 .

Case 3. The elements of the first two or the last two layers have the same color, say the first two layers are red and Cases 1 and 2 do not hold. Since last three layers contain both red and blue elements, let $M$ be a set of size at least 6 that is red. If $a, b, c \in M$, then $\emptyset,\{a\},\{b\},\{c\}, M$ form a monochromatic configuration $\mathcal{A}$.

Case 4. None of the Cases 1, 2, 3 hold. Let $\emptyset$ be red. Then, w.l.o.g. $\{1\}$ is blue and by pigeonhole principle $\{1,2\},\{1,3\},\{1,4\},\{1,5\}$ are all of the same color $t \in$ \{red, blue $\}$. If either [8] or $[8] \backslash\{i\}$ is of color $t, i=2,3,4,5$, then we have a monochromatic configuration $\mathcal{A}$. Otherwise $[8],[8] \backslash\{2\},[8] \backslash\{3\},[8] \backslash\{4\}, B$, where $B \in\{\emptyset,\{1\}\}$ form a monochromatic configuration $\mathcal{A}$.

It remains to show that if there is a monochromatic configuration $\mathcal{A}=\left\{A_{1}, A_{2}, A_{3}, A_{4}, A_{5}\right\}$ then there is a monochromatic copy of $Q_{3}$. Assume w.l.o.g. that $\left|A_{5} \backslash A_{i}\right| \geq 5$ for $i=2,3,4$. Further assume that $A_{i}=A \cup\{i\}$, for $i=2,3,4$ and some $A$. Consider $B_{k}=\left\{S: A \cup\{i, j\} \subseteq S \subseteq A_{5} \backslash\{k\}\right\}$ for $\{i, j, k\}=\{2,3,4\}$. Then each $B_{k}, k=2,3,4$ is a copy of $Q_{3}$. If at least one of this copies is monochromatic, we are done. Otherwise each $B_{k}$ has a red element, $C_{k}$. Then $A_{1}, A_{2}, A_{3}, A_{4}, C_{1}, C_{2}, C_{3}, A_{5}$ form a monochromatic copy of $Q_{3}$.

Proof of Theorem 1 (vi). Here, we do not try to optimize the constant 3. In fact, it could be replaced with $1+\epsilon$ for any positive $\epsilon$. Let $N=n+(n+1) 2 \log n$. Let $[N]=X_{0} \cup X_{1} \cup \cdots \cup X_{n+1}$, where $X_{i}$ 's are pairwise disjoint, $\left|X_{0}\right|=n$ and $\left|X_{i}\right|=m, i=1, \ldots n+1$, where $m=2 \log n$. We drop floors and ceilings here and assume that $m$ is even. Color subsets of $[N]$ with red and blue such that a set gets color red with probability $1 / 2$ and the sets are colored independently. For a set $S \subseteq X_{0}$, 
let $\mathcal{F}(S)=\left\{S \cup X_{1} \cup \cdots \cup X_{|S|} \cup X: X \subset X_{|S|+1},|X|=m / 2\right\}$. The probability that $\mathcal{F}(S)$ has only blue sets is $2^{-\left(\begin{array}{c}m \\ m / 2\end{array}\right)}$. The probability that each $\mathcal{F}(S)$ has a red set for each $S$ is thus at least $p(n)=1-2^{n} 2^{-\left(\begin{array}{c}m \\ m / 2\end{array}\right)}$. Here, we use a simple union bound and the fact that there are $2^{n}$ choices for $S$. Since $2^{n} 2^{-\left(\begin{array}{c}m \\ m / 2\end{array}\right)}=2^{n-\left(\begin{array}{c}m \\ m / 2\end{array}\right)} \leq 2^{n-n-\log n} \underset{n \rightarrow \infty}{\longrightarrow} 0$, we have that $p(n) \underset{n \rightarrow \infty}{\longrightarrow} 1$. Therefore, asymptotically almost surely each $\mathcal{F}(S)$ has a red set $F_{S}$. In that case $\left\{F_{S}: S \subseteq X_{0}\right\}$ is a copy of $Q_{n}$.

\section{Embeddings of $Q_{n}$ into $Q_{N}$.}

In this section we define two bijections between the set of copies of $Q_{n}$ in $Q_{N}$ and sets of other combinatorial objects. We use these bijections to prove Theorem 4 and to make some general observations about Ramsey numbers for Boolean lattices.

\subsection{Bijection from the set of good sequences}

Let $f: 2^{[n]} \rightarrow 2^{[N]}$ be an embedding. Consider the sequence of sets $\left(U_{1}(f), \ldots, U_{N}(f)\right)$ with

$$
U_{j}(f)=\left\{S \in 2^{[n]}: j \in f(S)\right\}, \quad j \in[N] .
$$

We call this the characteristic vector of the embedding $f$. An example is shown in Figure 2. We call a sequence of upper-closed sets from $2^{[n]}$ good if each of $(\{i\})^{+}, i=1, \ldots, n$ appear in that sequence. Note that some $U_{j}$ s could be empty. If, for example $U_{3}=\{1\}^{+}, U_{7}=\{3\}^{+}$, and $U_{8}=\{2\}^{+}$in an
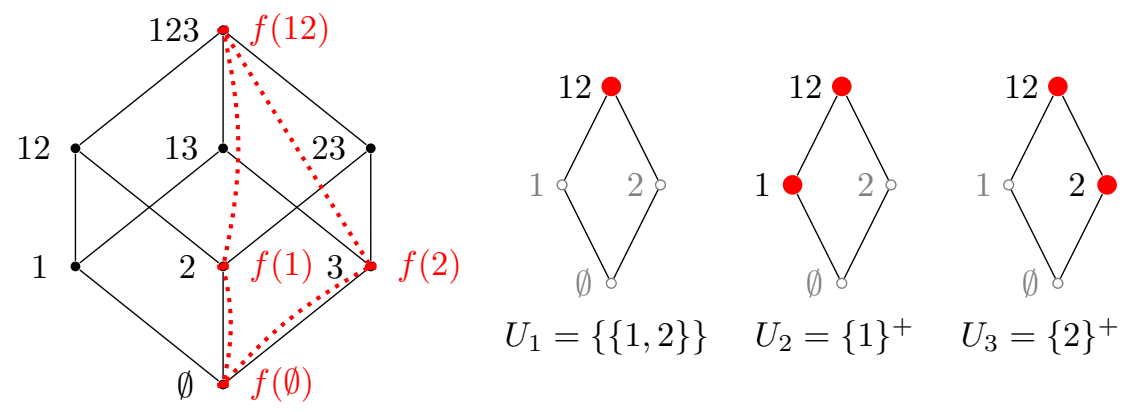

Figure 2: Example for an embedding $f$ of $Q_{2}$ into $Q_{3}$ with corresponding characteristic vector $\left(U_{1}, U_{2}, U_{3}\right)$.

embedding $f$ from $2^{[3]}$ to $2^{[9]}$, then any subset of $\{3,7,8\}$ is equal to $f(S) \cap\{3,7,8\}$, for some $S$. For example $\{7,8\} \subseteq f(\{3,2\})$ since $3 \notin f(\{3,2\})$, it follows that $f(\{3,2\}) \cap\{3,7,8\}=\{7,8\}$.

Theorem 8. There is a bijection between the set of embeddings of $2^{[n]}$ into $2^{[N]}$ and the set of good sequences of $N$ upper-closed sets from $2^{[n]}$. The bijective mapping assigns each such embedding its characteristic vector.

Proof. Claim 1 Let $\left(U_{1}, \ldots, U_{N}\right)$ be the characteristic vector of an embedding $f: 2^{[n]} \rightarrow 2^{[N]}$. Then for each $j=1, \ldots, N$, the set $U_{j}$ is upper closed and for each $i=1, \ldots, n$ there is a $U_{j}$ such that $U_{j}=\{i\}^{+}$. 
To see that each $U_{j}$ is upper closed, consider $S \in U_{j}$ and $S^{\prime} \supseteq S$. Then $j \in f(S)$, and $f(S) \subseteq f\left(S^{\prime}\right)$. Thus $j \in f\left(S^{\prime}\right)$, and so $S^{\prime} \in U_{j}$. To prove the second statement, note that for any $i \in[n]$ we have $\{i\} \nsubseteq[n] \backslash\{i\}$. Thus $f(\{i\}) \nsubseteq f([n] \backslash\{i\})$, implying that there is $j$ such that $j \in f(\{i\}) \backslash f([n] \backslash\{i\})$. Now consider any $S \subseteq[n]$. If $i \in S$, then $\{i\} \subseteq S$, so $j \in f(\{i\}) \subseteq f(S)$. If $i \notin S$, then $S \subseteq[n] \backslash\{i\}$, and because of $f(S) \subseteq f([n] \backslash\{i\})$ and $j \notin f([n] \backslash\{i\})$ we conclude $j \notin f(S)$. Thus $i \in S \Leftrightarrow j \in f(S)$. By definition we also have $j \in f(S) \Leftrightarrow S \in U_{j}$ and $i \in S \Leftrightarrow S \in\{i\}^{+}$. Thus $S \in\{i\}^{+} \Leftrightarrow S \in U_{j}$. This implies that $U_{j}=\{i\}^{+}$, proving Claim 1 .

Claim 2 If $\left(U_{1}, \ldots, U_{N}\right)$ is a sequence of upper closed sets and all $\{i\}^{+}$occur among the $U_{j} \mathrm{~s}$, for $i=1, \ldots, n$, then the function $f$ with $f(S)=\left\{j: S \in U_{j}\right\}$ is an embedding of $Q_{n}$ into $Q_{N}$. Moreover, $\left(U_{1}, \ldots, U_{N}\right)$ is the characteristic vector of $f$.

First we verify that $f$ is indeed an embedding. If $S \subseteq T$ then since $U_{j}$ 's are upper closed sets, if $S \in U_{j}$ then $T \in U_{j}$, so $f(S) \subseteq f(T)$. On the other hand, if $S \nsubseteq T$ then there is $i \in S \backslash T$. Let $j$ be an index such that $\{i\}^{+}=U_{j}$. Then $j \in f(S) \backslash f(T)$ and thus $f(S) \nsubseteq f(T)$. Now, to check that $\left(U_{1}, \ldots, U_{N}\right)$ is the characteristic vector of $f$, recall that $U_{j}(f)=\{S \subseteq[n]: j \in f(S)\}$. On the other hand $f(S)=\left\{j: S \in U_{j}\right\}$, so $U_{j}=\{S \subseteq[n]: j \in f(S)\}$. Thus $U_{j}=U_{j}(f)$, for all $j \in[N]$. This proves Claim 2.

Claim 3 If $f$ and $g$ are distinct embedding of $2^{[n]}$ into $2^{[N]}$ then they have distinct characteristic vectors, i.e., $\left(U_{1}(f), \ldots, U_{N}(f)\right) \neq\left(U_{1}(g), \ldots, U_{N}(g)\right)$.

Since $f \neq g$ we have $S \subseteq[n]$ with $f(S) \neq g(S)$, i.e., there is, without loss of generality $i \in f(S) \backslash g(S)$. Thus $S \in U_{i}(f)$, but $S \notin U_{i}(g)$, which proves Claim 3 .

Claims 1, 2, and 3 show that the characteristic vector provides the desired bijection.

\subsection{Bijection from the set of unions using inclusion preserving map}

Theorem 9. A set $\mathcal{S} \subseteq 2^{[N]}$ forms a copy of $2^{[n]}$ if and only if there is $I \subseteq[N]$ of size $n$ and an inclusion preserving map $\phi: 2^{I} \rightarrow 2^{[N] \backslash I}$ such that $\mathcal{S}=\{Y \cup \phi(Y): Y \subseteq I\}$.

Proof. Assume that there are such $I$ and $\phi$, so that $\mathcal{S}=\{Y \cup \phi(Y): Y \subseteq I\}$. We need to show that $\mathcal{S}$ induces a copy of $2^{[n]}$ in $2^{[N]}$. We shall show that $f: 2^{I} \rightarrow 2^{[N]}$, where $f(Y)=Y \cup \phi(Y)$ is an embedding. For this we need to observe that for $Y, Y^{\prime} \subseteq I$ we have $Y \subseteq Y^{\prime}$ iff $Y \cup \phi(Y) \subseteq Y^{\prime} \cup \phi\left(Y^{\prime}\right)$. Indeed, if $Y \subseteq Y^{\prime}$, then since $\phi$ is inclusion preserving, $\phi(Y) \subseteq \phi\left(Y^{\prime}\right)$, so $Y \cup \phi(Y) \subseteq Y^{\prime} \cup \phi\left(Y^{\prime}\right)$. If $Y \cup \phi(Y) \subseteq Y^{\prime} \cup \phi\left(Y^{\prime}\right)$, and $Y, Y^{\prime} \subseteq I, \phi(Y), \phi\left(Y^{\prime}\right) \subseteq[N] \backslash I$, we have that $Y \subseteq Y^{\prime}$.

The other way around, assume that $\mathcal{S}$ is a copy of $2^{[n]}$ in $2^{[N]}$ obtained as a result of an embedding $f$. From Theorem 8 , we know that the characteristic vector $\left(U_{1}, \ldots, U_{N}\right)$ of $f$ is good, i.e., there is an index set $I$ of size $n$ such that $U_{g(i)}=\{i\}^{+}$for $i \in[n]$ and $g$ is a bijection from $[n]$ to $I$. This means that $\{f(S) \cap I: S \subseteq[n]\}=2^{I}$. Since $|\mathcal{S}|=2^{|I|}$, there is for each $Y \subseteq I$ a unique $\phi(Y) \subseteq[N] \backslash I$ such that some set $S \in \mathcal{S}$ is equal to $Y \cup \phi(Y)$. It remains to show that $\phi$ is inclusion preserving. Assume that for some $Y, Y^{\prime} \subseteq I, Y \subseteq Y^{\prime}$ and $\phi(Y) \nsubseteq \phi\left(Y^{\prime}\right)$. Then the corresponding sets $Y \cup \phi(Y)$ and $Y^{\prime} \cup \phi\left(Y^{\prime}\right)$ are not comparable. Thus the number of comparable pairs is $\mathcal{S}$ is strictly less that the number of comparable pairs in $2^{I}$. This is a contradiction since $\mathcal{S}$ forms an copy of $2^{I}$. 


\subsection{Counting copies of $Q_{n}$}

Proof of Theorem 5. It follows from Theorem 8, that the number $e(n, N)$ of copies of $Q_{n}$ in $Q_{N}$ is equal to the number of good sequences. Each upper closed set is uniquely determined by an antichain of its minimal elements. For the upper bound, observe that there are $\left(\begin{array}{l}N \\ n\end{array}\right)$ ways to choose the positions of $\{1\}^{+}, \ldots,\{n\}^{+}$in the sequence. There are $n$ ! ways to place those in these positions. The remaining $N-n$ positions could be occupied by any upper closed set, so there are $a(n)^{N-n}$ ways to choose them. All together there are at most $\left(\begin{array}{c}N \\ n\end{array}\right) n ! a(n)^{N-n}=\frac{N !}{(N-n) !} a(n)^{N-n}$ ways to form such a good sequence. For the lower bound, we count only the good sequences with each $\{i\}^{+}$appearing exactly once. There are again $\frac{N !}{(N-n) !}$ ways to place $\{1\}^{+}, \ldots,\{n\}^{+}$in $n$ positions in the sequence and there are $(a(n)-n)^{N-n}$ ways to place upper sets different from these in the remaining $N-n$ positions.

Note that one could provide an exact formula for $e(N, n)$ in terms of $a(n)$ by counting the words of length $N$ over the alphabet $[a(n)]$, containing each of the letters $1,2, \ldots, n$ at least once.

Finally, to prove the last statement of the Theorem, observe that $a(n)=2^{\left(\left\lfloor\frac{n}{2}\right\rfloor\right)(1+O(\log n / n))}$, see [10], and $\frac{N !}{(N-n) !} \leq N^{n}=2^{n \log N}$.

\section{Multicolor Ramsey numbers}

Proof of Theorem 6. Consider a coloring $c$ of $Q_{N}$ in $k$ colors and having no monochromatic copy of $P$. Then each color class contains no copy of $P$ and thus Lubell mass of each color class $C_{i}$ is $\ell\left(C_{i}\right) \leq \lambda^{*}(P)$. Now, from the definition $\ell\left(2^{[N]}\right)=\sum_{i=1}^{k} \ell\left(C_{i}\right) \leq k \lambda^{*}(P)$. On the other hand, $\ell\left(2^{[N]}\right)=\sum_{i=0}^{N}\left(\begin{array}{c}N \\ i\end{array}\right)\left(\begin{array}{c}N \\ i\end{array}\right){ }^{-1}=N+1$. Thus $N+1 \leq k \lambda^{*}(P)=\Theta(k)$.

To show that $R_{k}(P) \geq \Omega(k)$, consider a layered copy of $Q_{k-1}$ with each layer of own color. Then each color class is an antichain, thus does not contain a copy of $P$. So, $R_{k}(P) \geq k$.

\section{Boolean algebras}

While Gunderson, Rödl, and Sidorenko [7] consider a multicolor Ramsey problem for Boolean algebras when the dimension of the desired monochromatic Boolean lattice is fixed and the number of colors grows, we consider a 2-colored case. Note that Layered Lemma 2 immediately gives a very large upper bound on $R_{\mathrm{Alg}}(n)$ in terms of repeated application of hypergraph Ramsey's Theorem and Hilbert's Theorem. Indeed, we know from Lemma 2 that sufficiently large 2-colored Boolean lattice contains a layered Boolean algebra $B$ of dimension $N^{\prime}$ with sets in layer $i$ of size $i$. Consider a coloring $c^{\prime}$ of $\left\{0,1, \ldots, N^{\prime}\right\}$, where $c^{\prime}(i)$ is the color of elements in layer $i, i=0, \ldots, N^{\prime}$. If $N^{\prime}>h(n, 2)$ then there is a monochromatic, say red, Hilbert cube of dimension $n$ in $c^{\prime}$, say $\left\{x_{0}+\sum_{i \in I} x_{i}: I \subseteq[n]\right\}$. This means that all subsets of $S_{n}$ of sizes $x_{0}, x_{0}+x_{1}, x_{0}+x_{2}, \ldots, x_{0}+x_{n}, x_{0}+x_{1}+x_{2}, \ldots, x_{0}+x_{1}+\cdots+x_{n}$ are red. Pick disjoint subsets $X_{0}, X_{1}, \ldots, X_{n}$ of $B$ of sizes $x_{0}, \ldots, x_{n}$ respectively. We can do this since $N^{\prime} \geq x_{0}+x_{1}+\cdots+x_{n}$. Then these sets generate a monochromatic Boolean algebra of dimension $n$.

Proof of Theorem \%. To get the first expression in the upper bound, consider a bound on $b(N, n)$, the largest number of elements in $Q_{N}$ not containing a Boolean algebra of dimension $n$, given in [7]:

$$
b(N, n) \leq 10^{n} 2^{-\frac{1}{2^{n-1}}} n^{n-\frac{1}{2^{n}}} \cdot N^{-\frac{1}{2^{n}}} 2^{N} .
$$

Thus if $N=2^{2^{n+1} n \log n}$ then there is a color class of size at least $2^{N} / 2>b(N, n)$. Therefore there is a monochromatic Boolean algebra of dimension $n$. 
To get the second expression in the upper bound, we use the ideas from [7] again. Consider a coloring $c$ of $Q_{N}$. Split [N] into $n$ pairwise disjoint sets $N_{1}, \ldots, N_{n}$ of almost equal sizes, further consider $\mathcal{C}_{i}$ to be a longest chain in a family of subsets of $N_{i}, i=1, \ldots, n$. Consider a complete $n$-uniform $n$-partite hypergraph with parts $\mathcal{C}_{i}, i=1, \ldots, n$. Color a hyperedge $\left\{X_{1}, X_{2}, \ldots, X_{n}\right\}$ with color $c\left(X_{1} \cup \cdots \cup X_{n}\right)$. If $N / n \geq R_{h}\left(K^{n}(2, \ldots, 2)\right)$ then we have a monochromatic $K^{n}(2, \ldots, 2)$ with partite sets $\left\{X_{1}, X_{1}^{\prime}\right\}, \ldots,\left\{X_{n}, X_{n}^{\prime}\right\}$, where $X_{i} \subseteq X_{i}^{\prime}$ and $X_{i}^{\prime} \cap X_{j}^{\prime}=\emptyset$ for $i \neq j$. This corresponds to a monochromatic Boolean algebra with sets $Y_{0} \cup\left\{\cup_{i \in I} Y_{i}: I \subseteq[n]\right\}$, where $Y_{0}=X_{1} \cup \cdots \cup X_{n}$ and $Y_{i}=X_{i}^{\prime} \backslash X_{i}, i=1, \ldots, n$.

For the lower bound, we use the layered coloring of $Q_{N}$ with $N=h(n, 2)-1$ in red and blue, so that the indices of blue layers form a set without affine cube of dimension $n$ and so do the indices of the red layers. It was noticed in [2], that it is easy to see that $h(n, 2) \geq 2^{c n}$.

\section{Conclusions}

In this paper, we initiated the study of Ramsey theory for copies of posets in Boolean lattices. Compared with at least exponential behavior of a similar Ramsey number for Boolean algebras, we obtain the bounds $2 n-1 \leq R\left(Q_{n}, Q_{n}\right) \leq n^{2}+2 n$. We give a tight asymptotical expression of the number of embeddings of $Q_{n}$ into $Q_{N}$ and show that the multicolor Ramsey number for posets is linear in the number of colors.

The question of determining the correct value for $R\left(Q_{n}, Q_{n}\right)$ remains one of the interesting here. The fact that $R\left(Q_{3}, Q_{3}\right) \in\{7,8\}$ suggests that neither of our bounds for $R\left(Q_{n}, Q_{n}\right)$ is tight. Another interesting question is how the colorings of $Q_{N}$, for $N=R\left(Q_{n}, Q_{n}\right)-1$ and no monochromatic copies of $Q_{n}$ look like. So far, for all lower bounds on Boolean lattices and Boolean algebras, only layered colorings were considered. Here, we presented a non-layered coloring of $Q_{6}$ with no monochromatic copy of $Q_{3}$. Note that any layered coloring of $Q_{6}$ has a monochromatic $Q_{3}$. For some additional Ramsey-type results in the Boolean lattice, see Walzer [22].

\section{Acknowledgements}

The authors thank Tom Trotter, Dwight Duffus, Kevin Milans, and David Gunderson for inspiring discussion. Particular thanks go to Dwight Duffus for bringing [7] to author's attention and mentioning different sorts of embeddings. The authors thank also Torsten Ueckerdt and Jonathan Rollin for discussions.

\section{References}

[1] M. Axenovich, A. Gyárfás, H. Liu, and D. Mubayi. "Multicolor Ramsey numbers for triple systems". In: Discrete Math. 322 (2014), pp. 69-77. DOI: 10.1016/j.disc.2014.01.004.

[2] T. C. Brown, P. Erdös, F. R. K. Chung, and R. L. Graham. "Quantitative forms of a theorem of Hilbert". In: J. Combin. Theory Ser. A 38.2 (1985), pp. 210-216. DOI: 10 . 1016/0097$3165(85) 90071-8$.

[3] D. Conlon, J. Fox, and B. Sudakov. "An improved bound for the stepping-up lemma". In: Discrete Appl. Math. 161.9 (2013), pp. 1191-1196. DOI: 10.1016/j.dam.2010.10.013.

[4] D. Conlon, J. Fox, and B. Sudakov. "Hypergraph Ramsey numbers". In: Journal of the American Mathematical Society 23.1 (2010), pp. 247-247. DOI: 10.1090/s0894-0347-09-00645-6. 
[5] D. Conlon, J. Fox, and B. Sudakov. "Ramsey numbers of sparse hypergraphs". In: Random Structures Algorithms 35.1 (2009), pp. 1-14. DOI: 10.1002/rsa. 20260.

[6] O. Cooley, N. Fountoulakis, D. Kühn, and D. Osthus. "Embeddings and Ramsey numbers of sparse $k$-uniform hypergraphs". In: Combinatorica 29.3 (2009), pp. 263-297. DOI: $10.1007 /$ s00493-009-2356-y.

[7] D. S. Gunderson, V. Rödl, and A. Sidorenko. "Extremal Problems for Sets Forming Boolean Algebras and Complete Partite Hypergraphs". In: Journal of Combinatorial Theory, Series A 88.2 (1999), pp. 342 -367. DOI: 10.1006/jcta.1999. 2973.

[8] D. Hilbert. "Ueber die Irreducibilität ganzer rationaler Functionen mit ganzzahligen Coefficienten". In: J. Reine Angew. Math. 110 (1892), pp. 104-129. DoI: 10.1515/crll.1892.110.104.

[9] H. Kierstead and W. T. Trotter. "A Ramsey theoretic problem for finite ordered sets". In: Discrete Mathematics 63.2-3 (1987), pp. 217-223. DOI: 10.1016/0012-365x(87)90009-4.

[10] D. Kleitman and G. Markowsky. "On Dedekind's problem: the number of isotone Boolean functions". In: Trans. Amer. Math. Soc. 213 (1975), pp. 373-390.

[11] A. Kostochka, D. Mubayi, and J. Verstraete. "Hypergraph Ramsey numbers: triangles versus cliques". In: J. Combin. Theory Ser. A 120.7 (2013), pp. 1491-1507. DoI: $10.1016 /$ j . jcta . 2013.04.009.

[12] J. H. van Lint and R. M. Wilson. A course in combinatorics. Second. Cambridge University Press, Cambridge, 2001, pp. xiv+602. DOI: 10.1017/CB09780511987045.

[13] A. Methuku and D. Pálvölgyi. "Forbidden hypermatrices imply general bounds on induced forbidden subposet problems". In: ArXiv e-prints (Aug. 2014). arXiv:1408.4093 [math.CO].

[14] A. Méroueh. "Lubell mass and induced partially ordered sets". In: ArXiv e-prints (2015). arXiv:1506. 07056.

[15] J. Nešetřil and V. Rödl. "Combinatorial partitions of finite posets and lattices - Ramsey lattices". In: Algebra Universalis 19.1 (1984), pp. 106-119. DOI: 10.1007/BF01191498.

[16] M. Paoli, W. T. Trotter, and J. W. Walker. "Graphs and orders in Ramsey theory and in dimension theory". In: Graphs and order. Vol. 147. NATO Adv. Sci. Inst. Ser. C Math. Phys. Sci. Reidel, Dordrecht, 1985, pp. 351-394.

[17] B. Patkos. "Induced and non-induced forbidden subposet problems". In: (2015). arXiv:1408.0899 [hep-th].

[18] F. P. Ramsey. "On a Problem of Formal Logic". In: Proceedings of the London Mathematical Society s2-30.1 (1930), pp. 264-286. DOI: 10.1112/plms/s2-30.1.264.

[19] W. T. Trotter. Combinatorics and Partially Ordered Sets: Dimension Theory. Baltimore: Johns Hopkins University Press, 1992.

[20] W. T. Trotter. "Embedding finite posets in cubes". In: Discrete Mathematics 12.2 (1975), pp. 165 -172. DOI: $10.1016 / 0012-365 X(75) 90031-X$.

[21] W. T. Trotter. "Ramsey theory and partially ordered sets". In: Contemporary Trends in Discrete Mathematics: From DIMACS and DIMATIA to the Future (1999), pp. 337-348.

[22] S. Walzer. "Ramsey Variant of the 2-Dimension of Posets". In: Master Thesis (2015).

[23] D. B. West. Introduction to Graph Theory. 2nd ed. Prentice Hall, Sept. 2000. 